\title{
Construction of commutative and associative operations by paving
}

\author{
Martin Kalina ${ }^{1}$ Pavol Král ${ }^{2}$ \\ ${ }^{1}$ Slovak University of Technology in Bratislava \\ Faculty of Civil Engineering, Department of Mathematics \\ Radlinského 11, Sk-810 05 Bratislava, Slovakia \\ e-mail: kalina@math.sk \\ ${ }^{2}$ Dept. of Quantitative Methods and Information Systems \\ Faculty of Economics, Matej Bel University \\ Tajovského 10, Sk-975 90 Banská Bystrica \\ Slovakia \\ e-mail: pavol.kral@umb.sk
}

\begin{abstract}
Paving is a method for constructing new operations from a given one. We will show that this method can be used to construct associative, commutative and monotone operations from particular given operations (from basic 'paving stones'). We will discuss properties of the resulting operations by considering different cases of the 'paving stones' and the starting position of paving. Finally, we will discuss the case when the basic 'paving stone' is a generated operation. We show that in this case we get by paving also a generated operation, just the generator is a two-place function. We show also an example of a non-representable uninorm which is strictly increasing in both variables on the open unit square.
\end{abstract}

Keywords: T-norm, uninorm, associative operation, paving, generated t-norm

\section{Introduction}

Associative operations such as t-norms, t-conorms, or uninorms as their common generalization, play an important role in fuzzy logic, in decision making, fuzzy control, and so on. Among these operations, those which are strictly increasing on $] 0,1\left[{ }^{2}\right.$, play an important role becuase of their cancelativity. The aim of this paper is to present a new construction possibility for associative operations. Several construction methods are already known. We show another method based on a so-called paving. Moreover we show that, using paving, it is possible to construct non-representable uninorms which are strictly increasing on $] 0,1\left[^{2}\right.$, see Example 1 at the end of this paper.

The idea of paving is the following. We split the unit interval into countably many disjoint subintervals $\left\{I_{i}\right\}_{i \in \mathcal{I}}$ (in such a way we split the unit square into countably many disjoint sub-rectangles $I_{i} \times I_{j}$ ). Then we take an operation $*:[0,1]^{2} \rightarrow[0,1]$, choose transformations $f_{i}: I_{i} \rightarrow[0,1]$ and we 'pave' the whole unit square (see Fig. 1 for a graphical schema of paving) by

$$
f_{i+j}^{[-1]}\left(f_{i}(x) * f_{j}(y)\right)
$$

where $f_{i+j}^{[-1]}$ is a kind of inverse (could be pseudoinverse or a quasi-inverse).

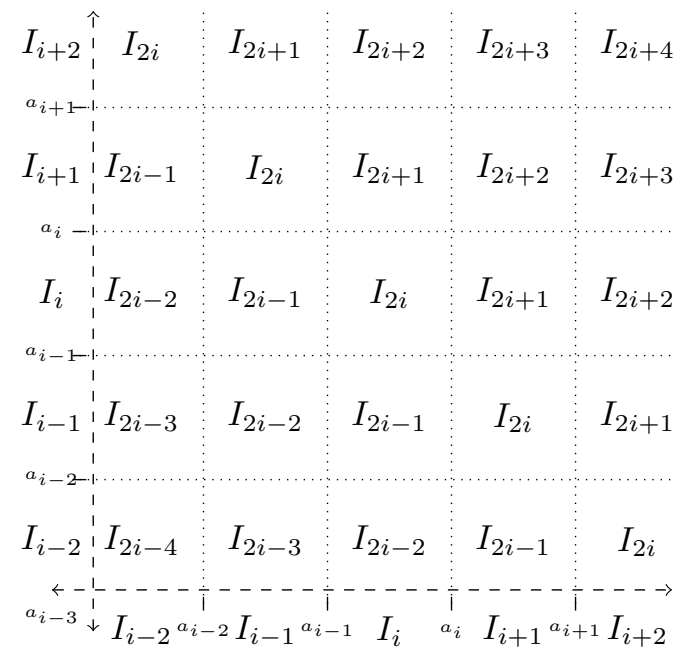

Figure 1: Graphical schema of paving

\section{Preliminaries}

We recall some known facts and notions to make the paper self-content.

A function $N:[0,1] \rightarrow[0,1]$ is said to be a negation if $N$ is decreasing and $N(0)=1, N(1)=0$.

A negation $N$ is said to be strong if it is involutive, i.e., if $N(N(x))=x$ for all $x \in[0,1]$.

Definition 1 (see, e.g., [3, 5]) A triangular norm $T:[0,1]^{2} \rightarrow[0,1]$ (t-norm for short) is a commutative, associative, increasing in both variables binary operation fulfilling the boundary condition $T(x, 1)=x$ for all $x \in[0,1]$. 
Remark 1 Note that, for a strong negation $N$, the $N$-dual operation to a t-norm $T$ defined by $S(x, y)=N(T(N(x), N(y)))$ is called a t-conorm. For more information, see, e.g., [3].

We will denote $T_{M}(x, y)=\min \{x, y\}$ and $S_{M}(x, y)=\max \{x, y\}$.

An operation $\tilde{T}:[0,1]^{2} \rightarrow[0,1]$ which is increasing in both variables, associative, commutative and $\tilde{T} \leq T_{M}$, is called a $t$-sub-norm.

Dually, $\tilde{S}:[0,1]^{2} \rightarrow[0,1]$ which is increasing in both variables, associative, commutative and $\widetilde{S} \geq$ $S_{M}$, is called a t-super-conorm.

T-sub-norms were introduced in [1] (see also [3]). T-super-conorms were introduced in [4].

For t-norms and t-conorms we have the following important inequalities.

Let $T:[0,1]^{2} \rightarrow[0,1]$ be a t-norm. Then $T(x, y) \leq$ $\min \{x, y\}$.

Let $S:[0,1]^{2} \rightarrow[0,1]$ be a t-conorm. Then $S(x, y) \geq \max \{x, y\}$.

Uninorms, as a generalization of triangular norms and conorms, were introduced by Yager and Rybalov in [6].

Definition 2 An associative, commutative and increasing operation $U:[0,1]^{2} \rightarrow[0,1]$ is called a uninorm, if there exists $e \in[0,1]$, called the neutral element of $U$, such that

$$
U(x, e)=x \quad \text { for all } x \in[0,1] .
$$

If $U(1,0)=0$ holds, $U$ is called conjunctive. If $U(1,0)=1$ holds, $U$ is called disjunctive. Conjunctive and disjunctive uninorms are dual to each other. For an arbitrary disjunctive uninorm $U$ and a strong negation $N$ its $N$-dual conjunctive uninorm is given by

$$
U_{N}^{d}(x, y)=N(U(N(x), N(y))) .
$$

Uninorms whose neutral element is in ]0,1[ will be called proper (to distinguish them from t-norms and/or t-conorms).

Definition 3 Let $*:[0,1]^{2} \rightarrow[0,1]$ be a commutative operation. Fix a value $a \in[0,1]$. We say that $x \in[0,1], x \neq a$, is an a-divisor if there exists $y \in[0,1], y \neq a$ such that

$$
x * y=a .
$$

Further, for increasing functions $f:[a, b] \rightarrow[c, d]$, functions $f^{\wedge}:[c, d] \rightarrow[a, b]$ and $f^{\vee}:[c, d] \rightarrow[a, b]$ were introduced in [2] as follows

$$
\begin{aligned}
& f^{\wedge}(y)=\sup \{z \in[a, b] ; f(z)<y\} \\
& f^{\vee}(y)=\inf \{z \in[a, b] ; f(z)>y\},
\end{aligned}
$$

where $\sup \emptyset=a, \inf \emptyset=b$.
Definition 4 ([2]) Let $f:[a, b] \rightarrow[c, d]$ be an increasing function. Arbitrary function $f^{*}:[c, d] \rightarrow$ $[a, b]$ is called a quasi-inverse of $f$ if it satisfies

$$
\begin{aligned}
& f\left(f^{*}(z)\right)=z \quad \text { for } z \in \operatorname{rng}(f), \\
& f^{\wedge} \leq f^{*} \leq f^{\vee} .
\end{aligned}
$$

Remark 2 Of course, $f^{\wedge}$ coincides with the wellknown pseudo-inverse $f^{(-1)}$. If $f$ is continuous with $\operatorname{rng}(f)=[c, d]$, both $f^{\wedge}$ as well as $f^{\vee}$, are special cases of quasi-inverses of $f$.

In the next lemma we give some important properties of $f^{\wedge}$ and $f^{\vee}$.

Lemma 1 Let $f:[a, b] \rightarrow[c, d]$ be a continuous increasing function with $\operatorname{rng}(f)=[c, d]$. Then:

(a) for all $z \in[c, d]$ we have $f\left(f^{\wedge}(z)\right)=z$ and also $f\left(f^{\vee}(z)\right)=z$,

(b) all $x \in[a, b]$ yield $f^{\wedge}(f(x)) \leq x$ and $f^{\vee}(f(x)) \geq$ $x$.

\section{Paving as a construction method}

Our intention is to construct an operation $\odot$ : $[0,1]^{2} \rightarrow[0,1]$ which is (not necessarily strictly) increasing in both variables, commutative and associative. We split the unit interval $[0,1]$ into countably many sub-intervals by choosing a sequence of their end-points. For a technical reason we will need the set of indices to be closed under addition. For this reason we will distiguish five possibilities. The set of indices will be denoted by $\mathcal{J}$. We set:

1. $0=a_{0}<a_{1}<a_{2}<\cdots<a_{n}<\cdots<a_{\infty}=1$,

2. $0=a_{-1}<a_{0}<a_{1}<\cdots<a_{n}<\cdots<a_{\infty}=1$,

3. $0=a_{-\infty}<\cdots<a_{-n}<a_{-n+1}<\cdots<a_{-2}<$ $a_{-1}=1$,

4. $0=a_{-\infty}<\cdots<a_{-n}<a_{-n+1}<\cdots<a_{-1}<$ $a_{0}=1$,

5. $0=a_{-\infty}<\cdots<a_{-n}<\cdots<a_{-1}<a_{0}<$ $a_{0}<a_{1}<\cdots<a_{n}<\cdots<a_{\infty}=1$.

For each interval $I_{i}=\left[a_{i-1}, a_{i}\right]$ we will have a monotone transformation $f_{i}: I_{i} \rightarrow[0,1]$. We will assume that $f_{i}\left(a_{i-1}\right)=0$ and $f_{i}\left(a_{i}\right)=1$. Moreover, we will assume continuity of all functions $f_{i}$.

Yet, $a_{i}$ belongs to two different intervals. This would cause problems. For this reasons we will consider semi-open intervals. We introduce the following notation:

$$
\begin{aligned}
I_{i}^{<} & =\left[a_{i-1}, a_{i}[,\right. \\
I_{i}^{>} & \left.=] a_{i-1}, a_{i}\right] .
\end{aligned}
$$

The basic idea of paving is hidden in the following two formulae which will be used alternatively. We choose an operation $*:[0,1]^{2} \rightarrow[0,1]$ which is isotone in both variables, commutative and associative. Then, for $i, j \in \mathcal{J}, x \in I_{i}^{<}$and $y \in I_{j}^{<}\left(x \in I_{i}^{>}\right.$ and $y \in I_{j}^{>}$, alternatively) we define

$$
x * \vee y=f_{i+j}^{\vee}\left(f_{i}(x) * f_{j}(y)\right)
$$


and

$$
x *^{\wedge} y=f_{i+j}^{\wedge}\left(f_{i}(x) * f_{j}(y)\right) .
$$

Formulae (5) and (6) directly imply that both operations, $*^{\vee}$ and $*^{\wedge}$, are isotone and commutative (when properly defined on the border). Let us discuss the associativity of $*^{\vee}$ and $*^{\wedge}$.

\subsection{Associativity}

We know already that, for continuous $f_{i}$ we have $f_{i}\left(f_{i}^{\vee}(x)\right)=x$ as well as $f_{i}\left(f_{i}^{\wedge}(x)\right)=x$. This means that if we use left-closed intervals, the operation $* \vee$ is associative if

$$
f_{i+j}^{\vee}\left(f_{i}(x) * f_{j}(y)\right) \in I_{i+j}^{<}
$$

for all $x \in I_{i}^{<}$and $y \in I_{j}^{<}$, and the same condition has to be fulfilled also for the operation $*^{\wedge}$ (and analogically we could treat the situation with rightclosed intervals). This gives the following propositions.

Proposition 1 Let the operation $*^{\vee}:[0,1]^{2} \rightarrow$ $[0,1]$ be defined by (5) for $x \in I_{i}^{<}$and $y \in I_{j}^{<}$, where $i, j \in \mathcal{J}$. It is associative on the open unit square ] $0,1\left[{ }^{2}\right.$ if one of the following conditions is fulfilled.

1. For all $f_{i}: I_{i}^{<} \rightarrow[0,1]$ and all $\left.z \in\right] a_{i-1}, a_{i}[$ we have $f_{i}(z)<1$, and $*$ has no 1 -divisors.

2. For each $f_{i}$ there exists $\left.z_{i} \in\right] a_{i-1}, a_{i}$ [ such that $f_{i}\left(z_{i}\right)=1$.

Proposition 2 Let the operation $*^{\vee}:[0,1]^{2} \rightarrow$ $[0,1]$ be defined by (5) for $x \in I_{i}^{>}$and $y \in I_{j}^{>}$, where $i, j \in \mathcal{J}$. It $i s$ associative on the open unit square $] 0,1\left[^{2}\right.$ if the following conditions is fulfilled.

1. For all $f_{i}: I^{>} \rightarrow[0,1]$ and all $\left.z \in\right] a_{i-1}, a_{i}[$ we have $f_{i}(z)>0$,

2. * has no 0-divisors.

Dually, we get the following.

Proposition 3 Let the operation $*^{\wedge}:[0,1]^{2} \rightarrow$ $[0,1]$ be defined by (6) for $x \in I_{i}^{>}$and $y \in I_{j}^{>}$, where $i, j \in \mathcal{J}$. It is associative on the open unit square ] $0,1[2$ if one of the following conditions is fulfilled.

1. For all $f_{i}$ and all $\left.z \in\right] a_{i-1}, a_{i}\left[\right.$ we have $f_{i}(z)>$ 0 , and * has no 0-divisors.

2. For each $f_{i}$ there exists $\left.z_{i} \in\right] a_{i-1}, a_{i}[$ such that $f_{i}\left(z_{i}\right)=0$.

Proposition 4 Let the operation $*^{\wedge}:[0,1]^{2} \rightarrow$ $[0,1]$ be defined by $(6)$ for $x \in I_{i}^{<}$and $y \in I_{j}^{<}$, where $i, j \in \mathcal{J}$. It is associative on the open unit square ] $0,1\left[{ }^{2}\right.$ if the following conditions are fulfilled.

1. For all $f_{i}$ and all $\left.z \in\right] a_{i-1}, a_{i}\left[\right.$ we have $f_{i}(z)<$ 1 ,

2. * has no 1-divisors.

A triple (operation, set of semi-open intervals, set of functions), where the operation is either $*^{\vee}$ or $*^{\wedge}$, will be called admissible if the corresponding operation got by paving is associative.

\subsection{Basic types of operations constructed via paving}

In this part we will show under which conditions we can construct by paving a t-norm, t-sub-norm, t-conorm, t-super-conorm, or proper uninorm. Formulae (5) and (6) immediately imply the following comparison of constructed operations with $T_{M}$ and $S_{M}$.

Proposition 5 Let $\mathcal{J}$ be an index set and $\tilde{*}=*^{\wedge}$ or $\tilde{*}=* \vee$ be a binary operation defined by (5) or (6), respectively, from a binary operation $*$ such that $\left(\tilde{*},\left\{I_{i}\right\}_{i \in \mathcal{J}},\left\{f_{i}\right\}_{i \in \mathcal{J}}\right)$ is an admissible triple and intervals $I_{i}$ are either left- or right-open. Then for $x \in I_{i}$ and $y \in I_{j}$ we have

$x \tilde{*} y \begin{cases}\geq S_{M}(x, y) & \text { if } i, j \geq 1, \\ \leq T_{M}(x, y) & \text { if } i, j \leq-1, \\ \in\left[T_{M}(x, y), S_{M}(x, y)\right] & \text { if } \min \{i, j\} \leq-1 \\ & \text { and } \max \{i, j\} \geq 1 .\end{cases}$

\subsubsection{Case when indices in $\mathcal{J}$ are non-negative}

Because of Proposition 5 we distinguish two cases when $0 \notin \mathcal{J}$ and $0 \in \mathcal{J}$.

Theorem 1 Assume $\mathcal{J}=\{1,2,3, \ldots\}$. Let $*_{1}^{\wedge}$ and $*_{2}^{\vee}$ be binary operations defined by (5) or (6), respectively, from a binary operation * such that $\left(*_{1}^{\wedge},\left\{I_{i}\right\}_{i \in \mathcal{J}},\left\{f_{i}\right\}_{i \in \mathcal{J})}\right.$ as well as $\left(*_{2}^{\vee},\left\{I_{i}\right\}_{i \in \mathcal{J}},\left\{f_{i}\right\}_{i \in \mathcal{J}}\right)$, are admissible triples for intervals $I_{i}$ being either left-open or right-open for $i \geq 2$ and $I_{1}$ is closed or right-open, respectively. Then

$$
x *_{1}^{\wedge} y= \begin{cases}f_{i+j}^{\wedge}\left(f_{i}(x) * f_{j}(y)\right) & \text { if } x \in I_{i}, y \in I_{j}, \\ 1 & \text { if } \max \{x, y\}=1,\end{cases}
$$

and

$$
x *_{2}^{\vee} y= \begin{cases}f_{i+j}^{\vee}\left(f_{i}(x) * f_{j}(y)\right) & \text { if } x \in I_{i}, y \in I_{j}, \\ 1 & \text { if } \max \{x, y\}=1,\end{cases}
$$

are t-super-conorms.

Theorem 2 Assume $\mathcal{J}=\{0,1,2, \ldots\}$. Let $*{ }_{3}^{\wedge}$ be a binary operation defined by (6) from an arbitrary $t$ super-conorm $*$ such that $\left(* \wedge,\left\{I_{i}^{>}\right\}_{i \in \mathcal{J}},\left\{f_{i}\right\}_{i \in \mathcal{J}}\right)$ is an admissible triple, where $I_{0}$ is a closed interval. Then

$x *_{3}^{\wedge} y= \begin{cases}f_{i+j}^{\wedge}\left(f_{i}(x) * f_{j}(y)\right) & \text { if } x \in I_{i}^{>}, y \in I_{j}^{>}, \\ 1 & \text { if } \max \{x, y\}=1, \\ f_{i+j}^{\wedge}\left(f_{i}(x) * f_{j}(y)\right) & \text { if } x \in I_{i}^{>}, y \in I_{j}^{>}, \\ & \text {and } \min \{x, y\}=0,\end{cases}$

is a t-super-conorm. Moreover, if all functions $f_{i}$ : $\left[a_{i-1}, a_{i}\right] \rightarrow[0,1]$ are bijections and $*$ is a t-conorm, then $* \hat{3}$ is a t-conorm. 
Theorem 3 Assume $\mathcal{J}=\{0,1,2, \ldots\}$. Let $*_{4}^{\wedge}$ be a binary operation defined by (6) from a binary operation $*$. Further assume that $*$ is a t-super-conorm without 1-divisors. Then $\left(*_{4}^{\wedge},\left\{I_{i}^{<}\right\}_{i \in \mathcal{J}},\left\{f_{i}\right\}_{i \in \mathcal{J}}\right)$ is an admissible triple and

$$
x *_{4}^{\wedge} y= \begin{cases}f_{i+j}^{\wedge}\left(f_{i}(x) * f_{j}(y)\right) & \text { if } x \in I_{i}^{<}, y \in I_{j}^{<}, \\ 1 & \text { if } \max \{x, y\}=1,\end{cases}
$$

is a t-super-conorm. Moreover, if $*$ is a t-conorm without 1 -divisors and all functions $f_{i}:\left[a_{i-1}, a_{i}\right] \rightarrow$ $[0,1]$ are bijections, $*_{3}^{\wedge}$ is a t-conorm.

Remark 3 (a) Because of Lemma 1(b), when $\mathcal{J}=$ $\{0,1,2, \ldots\}$ we can use only the operation $*^{\wedge}$, i.e., defined by formula (6), to construct t-superconorms (or t-conorms as special cases).

(b) In Theorems 2 and 3, to make 0 the neutral element of $*_{3}^{\wedge}$ and $*_{4}^{\wedge}$, respectively, (in case $*$ is a t-conorm) we need all functions $f_{i}$ to be bijective.

\subsubsection{Case when indices in $\mathcal{J}$ are non-positive}

This case is dual to the foregoing case with indices $\mathcal{J}$ being non-negative. Just everywhere instead of $\mathrm{t}$-conorm ( $\mathrm{t}$-super-conorm) we must use t-norm ( $\mathrm{t}$ sub-norm), instead of $*^{\wedge}$ the operation $*^{\vee}$ (and viceversa) and instead of left-open intervals it is necessary to take right-open intervals (and vice-versa).

\subsubsection{The index-set $\mathcal{J}=\mathbb{Z}$}

By Proposition 5 we get immediately that, for $\mathcal{J}=$ $\mathbb{Z}$, properties of arbitrary operation constructed by paving depend only on the behaviour of that operation in the area when one of the variables is in the interval $I_{0}$.

Theorem 4 Let $* \hat{5}$ be a binary operation defined by (6) from an arbitrary $t$-conorm * such that $\left(*^{\wedge},\left\{I_{i}^{>}\right\}_{i \in \mathbb{Z}},\left\{f_{i}\right\}_{i \in \mathbb{Z}}\right)$ is an admissible triple. Then

$$
x *{ }_{5}^{\wedge} y= \begin{cases}f_{i+j}^{\wedge}\left(f_{i}(x) * f_{j}(y)\right) & \text { if } x \in I_{i}^{>}, y \in I_{j}^{>}, \\ 1 & \text { if } \max \{x, y\}=1, \\ 0 & \text { if } \min \{x, y\}=0 \\ & \text { and } \max \{x, y\}<1,\end{cases}
$$

is an associative and commutative operation such that:

(S1) $x * \hat{5} y \geq S_{M}(x, y)$ for $\left.\left.(x, y) \in\right] a_{-1}, 1\right]^{2}$,

(S2) $* \hat{5}\left\lceil\left[0, a_{-1}\right]^{2}\right.$ is a t-sub-norm,

(S3) $T_{M}(x, y) \leq x * \hat{5} y \leq S_{M}(x, y)$ for $(x, y) \in$ $\left.\left.\left.\left.\left[0, a_{-1}\right] \times\right] a_{-1}, 1\right] \cup\right] a_{-1}, 1\right] \times\left[0, a_{-1}\right]$.

Dually we can formulate the following assertion.

Theorem 5 Let $*_{6}^{\vee}$ be a binary operation defined by (5) from an arbitrary t-norm * such that $\left(*^{\vee},\left\{I_{i}^{<}\right\}_{i \in \mathbb{Z}},\left\{f_{i}\right\}_{i \in \mathbb{Z}}\right)$ is an admissible triple. Then

$$
x *_{6}^{\vee} y= \begin{cases}f_{i+j}^{\vee}\left(f_{i}(x) * f_{j}(y)\right) & \text { if } x \in I_{i}^{<}, y \in I_{j}^{<}, \\ 1 & \text { if } \max \{x, y\}=1 \\ & \text { and } \min \{x, y\}>0, \\ 0 & \text { if } \min \{x, y\}=0,\end{cases}
$$

is an associative and commutative operation such that:

(C1) $*_{6}^{\vee}\left\lceil\left[a_{0}, 1\right]^{2}\right.$ is a t-super-conorm,

(C2) $x *_{6}^{\vee} y \leq T_{M}(x, y)$ for $(x, y) \in\left[0, a_{0}\left[{ }^{2}\right.\right.$,

(C3) $T_{M}(x, y) \leq x *_{6}^{\vee} y \leq S_{M}(x, y)$ for $(x, y) \in$ $\left[0, a_{0}\left[\times\left[a_{0}, 1\right] \cup\left[a_{0}, 1\right] \times\left[0, a_{0}[\right.\right.\right.$.

Operations with properties listed in Theorems 4 and 5 will be used in the next section to construct uninorms.

Definition 5 An associative, commutative and isotone operation $\odot:[0,1]^{2} \rightarrow[0,1]$ will be called a sub-uninorm if there exists an element $e \in[0,1]$ such that the following properties are fulfilled.

- $x \odot y \geq S_{M}(x, y)$ for $\left.\left.(x, y) \in\right] e, 1\right]^{2}$,

- $\odot \uparrow[0, e]^{2}$ is a t-sub-norm,

- $T_{M}(x, y) \leq x \odot y \leq S_{M}(x, y)$ for $(x, y) \in$ $[0, e] \times] e, 1] \cup] e, 1] \times[0, e]$.

A sub-uninorm $\odot$ is conjunctive (disjunctive) if $0 \odot$ $1=0(0 \odot 1=1)$. The element e will be called splitting.

Definition 6 An associative, commutative and isotone operation $\odot:[0,1]^{2} \rightarrow[0,1]$ will be called a super-uninorm if there exists an element $e \in[0,1]$ such that the following properties are fulfilled.

- $\odot[e, 1]^{2}$ is a t-super-conorm,

- $x \odot y \leq T_{M}(x, y)$ for $(x, y) \in[0, e]^{2}$,

- $T_{M}(x, y) \leq x \odot y \leq S_{M}(x, y)$ for $(x, y) \in$ $[0, e[\times[e, 1] \cup[e, 1] \times[0, e[$.

A super-uninorm $\odot$ is conjunctive (disjunctive) if $0 \odot 1=0(0 \odot 1=1)$. The element $e$ will be called splitting.

A. Mesiarová-Zemánková in [4] introduced the notion of a generalized uninorm $G$ which has an element $e \in[0,1]$ (called splitting). Generalized uninorms $G$ are operations such that $G \vdash[0, e]^{2}$ is a t-sub-norm and $G\left\lceil[e, 1]^{2}\right.$ is a t-super-conorm. The operation $* \hat{5}\left\lceil\left[a_{-1}, 1\right]^{2}\right.$ (S1)-(S3) from Theorem 4 is not a t-super-conorm and the operation $*_{6}^{\vee}\left\lceil\left[0, a_{0}\right]^{2}\right.$ from Theorem 5 is not a t-sub-norm.

Theorem 6 Let $* \hat{7}$ be a binary operation defined by (6) from a binary operation *. Further assume that $*$ is a t-conorm without 1 -divisors and all functions $f_{i}:\left[a_{i-1}, a_{i}\right] \rightarrow[0,1]$ are bijections. Then $\left(* \wedge,\left\{I_{i}^{<}\right\}_{i \in \mathbb{Z}},\left\{f_{i}\right\}_{i \in \mathbb{Z}}\right)$ is an admissible triple and

$$
x * \wedge \text { 과 } y= \begin{cases}f_{i+j}^{\wedge}\left(f_{i}(x) * f_{j}(y)\right) & \text { if } x \in I_{i}^{<}, y \in I_{j}^{<}, \\ 1 & \text { if } \max \{x, y\}=1, \\ 0 & \text { if } \min \{x, y\}=0 \\ & \text { and } \max \{x, y\}<1,\end{cases}
$$

is a proper disjunctive uninorm. 
Remark 4 (a) We could formulate a dual version of Theorem 6 replacing t-conorm without 1-divisors for a t-norm without 0-divisors and right-open intervals for left-open. The result would be again a proper uninorm.

(b) The operation $*_{5}^{\wedge}$ in Theorem 4 is a disjunctive sub-uninorm and the operation $*_{6}^{\wedge}$ in Theorem 5 is a conjunctive super-uninorm.

Theorems 4, 5, 6 are in fact reformulated results from part 3.2.2. Another possibility is to use a uninorm for the operation $*$.

Theorem 7 Let $*_{8}^{\wedge}$ be a binary operation defined by (6) from a proper uninorm *. Assume that all functions $f_{i}:\left[a_{i-1}, a_{i}\right] \rightarrow[0,1]$ are bijections. If * has no 1-divisors (no 0-divisors) then $\left(*_{8}^{\wedge},\left\{I_{i}^{<}\right\}_{i \in \mathbb{Z}},\left\{f_{i}\right\}_{i \in \mathbb{Z}}\right)$ is an admissible triple $\left(\left(*_{8}^{\wedge},\left\{I_{i}^{>}\right\}_{i \in \mathbb{Z}},\left\{f_{i}\right\}_{i \in \mathbb{Z}}\right)\right.$ is an admissible triple, respectively) and

$x *{ }_{8}^{\wedge} y= \begin{cases}f_{i+j}^{\wedge}\left(f_{i}(x) * f_{j}(y)\right) & \text { if } x \in I_{i}, y \in I_{j}, \\ 1 & \text { if } \max \{x, y\}=1, \\ 0 & \text { if } \min \{x, y\}=0 \\ & \text { and } \max \{x, y\}<1,\end{cases}$

where $I_{i}=I_{i}^{<}$or $I_{i}=I_{i}^{>}$, respectively, is a proper disjunctive uninorm.

\section{Modifications of paving}

Section 3 was devoted to describing the paving method as a method for construction of associative commutative and monotone operations. Now, we introduce some modifications of this method.

\subsection{Using 'halved paving stones'}

Let us consider the operation $*_{8}^{\wedge}$ constructed in Theorem 7. This is a uninorm, i.e., $*_{8}^{\wedge} \uparrow[0, e]^{2}$ is a t-norm (dually, $* \stackrel{\wedge}{\wedge}[e, 1]^{2}$ is a t-conorm). This t-norm (t-conorm) is constructed also by paving, just there is one difference. In an L-shaped area near the neutral element we use only 'halved' paving stones. Correctly expressed, $f_{0}:\left[a_{-1}, 1\right] \rightarrow[0, e]$. $\left(f_{0}:\left[0, a_{0}\right] \rightarrow[e, 1]\right.$ for the t-conorm). This gives the following assertion which we formulate only for the case of a t-norm.

Theorem 8 Set $\mathcal{J}=\{0,-1,-2, \ldots\}$. Let $*_{\hat{9}}^{\wedge}$ be a binary operation defined by (6) from a proper uninorm *. Assume that, for $i \leq-1$, all functions $f_{i}:\left[a_{i-1}, a_{i}\right] \rightarrow[0,1]$ are bijections and $f_{0}:\left[a_{-1}, 1\right] \rightarrow[0, e]$ is also bijective. Further denote $I_{0}^{<}=\left[a_{-1}, 1\right]$ and $\left.\left.I_{0}^{>}=\right] a_{-1}, 1\right]$. If $*$ has no 1 divisors (no 0 -divisors) then $\left(*_{9}^{\wedge},\left\{I_{i}^{<}\right\}_{i \in \mathcal{J}},\left\{f_{i}\right\}_{i \in \mathcal{J}}\right)$ is an admissible triple $\left(\left(*_{9}^{\wedge},\left\{I_{i}^{>}\right\}_{i \in \mathcal{J}},\left\{f_{i}\right\}_{i \in \mathcal{J}}\right)\right.$ is an admissible triple, respectively) and

$$
x * \hat{9} y= \begin{cases}f_{i+j}^{\wedge}\left(f_{i}(x) * f_{j}(y)\right) & \text { if } x \in I_{i}, y \in I_{j}, \\ 0 & \text { if } \min \{x, y\}=0\end{cases}
$$

where $I_{i}=I_{i}^{<}$or $I_{i}=I_{i}^{>}$, respectively, is a t-norm.

\subsection{Combining paving with ordinal sum-like construction}

In [4] an ordinal sum construction for systems of uninorms is described. We give here just the idea of the construction from [4]. For simplicity reasons we will consider only two proper uninorms $\left(\left\langle U_{k}, a_{k}, b_{k}, c_{k}, d_{k}\right\rangle \mid k \in\{1,2\}\right)$. Their ordinal sum is sketched on Fig. 2. Of course, the ordinal sum construction published in [4] is much more general. But for our purposes it is enough to consider the ordinal sum of two uninorms where values in rectangles $\left[a_{i}, b_{i}\right] \times\left[c_{i}, d_{i}\right]$ (and $\left[c_{i}, d_{i}\right] \times\left[a_{i}, b_{i}\right]$ ) are somehow transformed to $\left[a_{i}, b_{i}\right] \cup\left[c_{i}, d_{i}\right]$.

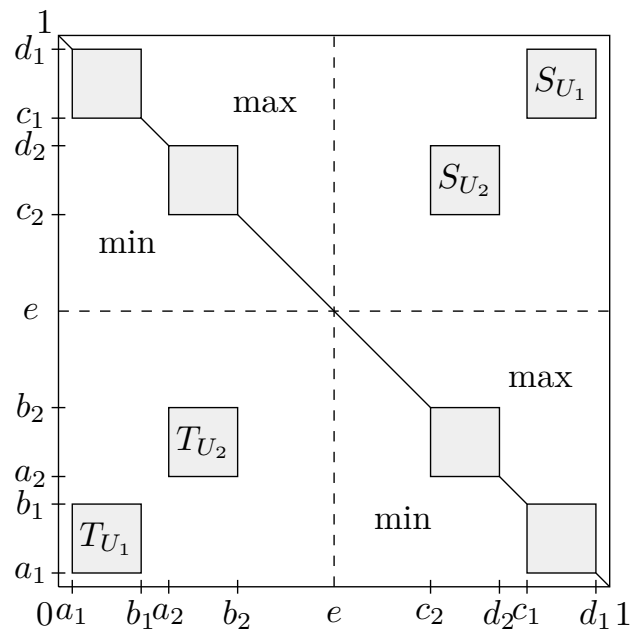

Figure 2: Ordinal sum of uninorms

Following the idea of the ordinal sum of uninorms we get the following construction of a uninorm from sub-uninorm (see Fig. 3).

Proposition 6 Let $\hat{*}:[0,1]^{2} \rightarrow[0,1]$ be a subuninorm with $e \in] 0,1[$ as its splitting element. Let us choose $\gamma \in] 0, s[$. Then, the operation $\odot$ : $[0,1]^{2} \rightarrow[0,1]$ defined by

$$
x \odot y= \begin{cases}\frac{\gamma}{e}\left(\frac{x \cdot e}{\gamma} \hat{*} \frac{y \cdot e}{\gamma}\right) & \text { if }(x, y) \in[0, \gamma]^{2}, \\ T_{M}(x, y) & \text { if }(x, y) \in[0, e]^{2} \backslash[0, \gamma]^{2}, \\ x \hat{*} y & \text { if }(x, y) \in] e, 1]^{2}, \\ S_{M}(x, y) & \text { if }(x, y) \in] \gamma, e] \times] e, 1] \\ & \text { or }(x, y) \in] e, 1] \times] \gamma, e], \\ \frac{x \cdot e}{\gamma} \hat{*} y & \text { if }(x, y) \in[0, \gamma] \times] e, 1] \\ \frac{\gamma}{e}\left(\frac{x \cdot e}{\gamma} \hat{*} y\right) & \text { and } \frac{x \cdot e}{\gamma} \hat{*} y>e, \\ & \text { if }(x, y) \in[0, \gamma] \times] e, 1] \\ x \hat{*} \frac{y \cdot e}{\gamma} & \text { if }(x, y) \in] e, 1] \times[0, \gamma] \\ & \text { and } x \hat{*} \frac{y \cdot e}{\gamma}>e, \\ \frac{\gamma}{e}\left(x \hat{*} \frac{y \cdot e}{\gamma}\right) & \text { if }(x, y) \in] e, 1] \times[0, \gamma] \\ & \text { and } x \hat{*} \frac{y \cdot e}{\gamma} \leq e,\end{cases}
$$

is a proper uninorm whose neutral element is e. 


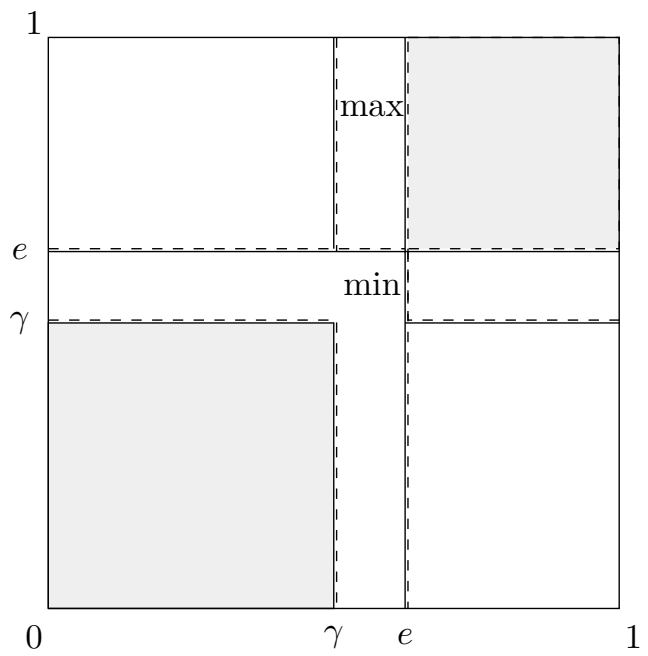

Figure 3: Ordinal sum-like construction of a uninorm from sub-uninorm

Dually, from a super-uninorm we can construct a uninorm in the following way (see Fig. 4).

Proposition 7 Let $\hat{*}:[0,1]^{2} \rightarrow[0,1]$ be a superuninorm with $e \in] 0,1[$ as its splitting element. Let us choose $\gamma \in]$ e, $1[$. Then, the operation $\odot$ : $[0,1]^{2} \rightarrow[0,1]$ defined by

$x \odot y= \begin{cases}x \hat{\odot} y & \text { if }(x, y) \in\left[0, e\left[{ }^{2},\right.\right. \\ h^{-1}(h(x) \hat{*} h(y)) & \text { if }(x, y) \in] \gamma, 1]^{2}, \\ S_{M}(x, y) & \text { if }(x, y) \in[e, 1]^{2} \backslash[\gamma, 1]^{2}, \\ T_{M}(x, y) & \text { if }(x, y) \in[0, e[\times[e, \gamma[ \\ & \text { or }(x, y) \in[e, \gamma[\times[0, e[, \\ x \hat{*} h(y) & \text { if }(x, y) \in[0, e[\times[\gamma, 1] \\ & \text { and } x \hat{*} h(y)<e, \\ h^{-1}(x \hat{*} h(y)) & \text { if }(x, y) \in[0, e[\times[\gamma, 1] \\ & \text { and } x \hat{*} h(y) \geq e, \\ h(x) \hat{*} y & \text { if }(x, y) \in[\gamma, 1] \times[0, e[ \\ & \text { and } h(x) \hat{*} y<e, \\ h^{-1}(h(x) \hat{*} y) & \text { if }(x, y) \in[\gamma, 1] \times[0, e[ \\ & \text { and } h(x) \hat{*} y \geq e,\end{cases}$

is a proper uninorm whose neutral element is e, and the transformation $h:[\gamma, 1] \rightarrow[e, 1]$ is defined by

$$
h(x)=(1-e) \frac{x-\gamma}{1-\gamma}+e .
$$

\section{Generated operation $*$}

Assume that the operation $*$ is generated and its additive generator is a function $g:[0,1] \rightarrow[-\infty, \infty]$. Then we can write

$$
x * y=g^{(-1)}(g(x)+g(y)),
$$

where $g$ is considered to be left-continuous and $g^{(-1)}$ is the pseudo-inverse. Of course, we can use also

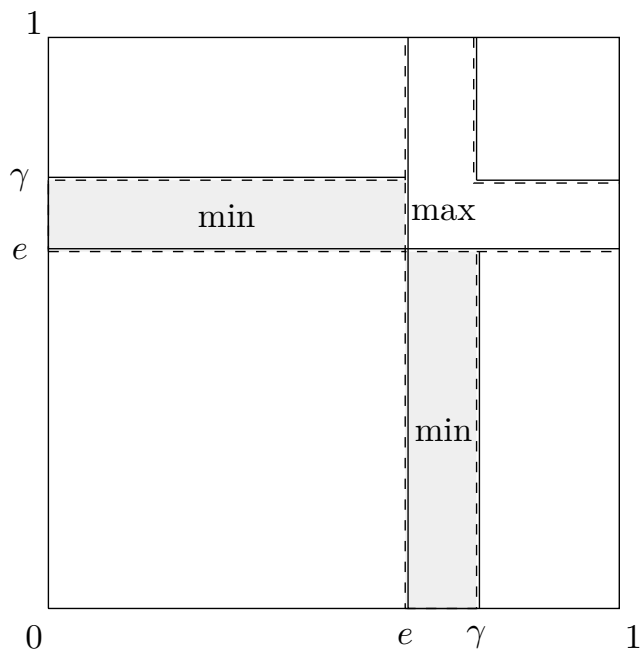

Figure 4: Ordinal sum-like construction of a uninorm from super-uninorm

right-continuous generator $g$, as well. Also if $g$ is a decreasing function, we can consider $-g$ instead. This means that the generator $g$ can be always taken as an increasing function which is left- or rightcontinuous. Hence, we can write

$x * y= \begin{cases}g^{\vee}(g(x)+g(y)) & \text { if } g \text { is left-continuous, } \\ g^{\wedge}(g(x)+g(y)) & \text { if } g \text { is right-continuous. }\end{cases}$

Thus, assume that $*$ is a generated operation with an additive generator $g$, and $\odot$ is a binary operation defined via paving from * such that $\left(\odot,\left\{I_{i}\right\}_{i \in \mathcal{J}},\left\{f_{i}\right\}_{i \in \mathcal{J}}\right)$ is an admissible triple. We extend $\mathcal{J}$ and the system of intervals $\left\{I_{i}\right\}_{i \in \mathcal{J}}$ in such a way that we add $\infty,-\infty$, or both values to the index set, if the indices are non-negative, non-positive, or $\mathcal{J}=\mathbb{Z}$, respectively. We set $I_{\infty}=\{1\}, I_{-\infty}=\{0\}$. We define function $\Gamma: \bigcup_{i \in \mathcal{J}}\{i\} \times I_{i} \rightarrow[-\infty, \infty]$ by

$$
\Gamma(i, x)=g\left(f_{i}(x)\right)
$$

which is an additive generator of $\odot$.

Example 1 Assume that $*=T_{P}$ (i.e., $*$ is the product t-norm).

(a) We can use $T_{P}$ to construct another t-norm (utilizing a theorem dual to Theorem 3) using a system of left-open intervals (for $\mathcal{J}=\{0,-1,-2,-3, \ldots\}$ ), e.g., $\left.\left.I_{i}=\right] 2^{i-1}, 2^{i}\right]$ and $I_{-\infty}=\{0\}$. For a generator $g:[0,1] \rightarrow[-\infty, 0]$ of $T_{P}$ we can take $g(x)=\ln x$. Yet, we need a system of bijections $\left.\left.f_{i}: I_{i} \rightarrow\right] 0,1\right]$ :

$$
f_{i}(x)= \begin{cases}\frac{x-2^{i-1}}{2^{i}-2^{i-1}} & \text { if } \left.x \in] 2^{i-1}, 2^{i}\right] \\ 0 & \text { if } x=0, \text { i.e. if } i=-\infty .\end{cases}
$$

If we define $\ln 0=-\infty$, we get the following generator of the operation $*^{\vee}$ constructed by paving from $T_{P}$ :

$$
\Gamma(i, x)=\ln f_{i}(x) .
$$

(b) We can use $T_{P}$ to construct a proper uninorm (utilizing a theorem dual to Theorem 6) using a 
system of left-open intervals

$$
I_{i}= \begin{cases}] \frac{1}{4}, \frac{3}{4}\right] & \text { for } i=0 \\ ] \frac{1}{2^{i-2}}, \frac{1}{2^{i-1}}\right] & \text { for } i \leq-1 \\ ] \frac{2^{i+1}-1}{2^{i+1}}, \frac{2^{i+2}-1}{2^{i+2}}\right] & \text { for } i \geq 1 \\ 1 & \text { for } i=\infty \\ 0 & \text { for } i=-\infty\end{cases}
$$

and a system of transformations $f_{i}$ :

$$
f_{i}(x)= \begin{cases}\frac{x-a_{i-1}}{a_{i}-a_{i-1}} & \text { for } i \in \mathbb{Z} \\ 1 & \text { for } i=\infty \\ 0 & \text { for } i=-\infty\end{cases}
$$

where $a_{i}$ is the right end-point of $I_{i}$. Then a generator of the corresponding uninorm constructed via paving is again

$$
\Gamma(i, x)=\ln f_{i}(x) .
$$

In fact, we get two different uninorms in this way a conjunctive and a disjunctive one depending on the convention $\infty-\infty=-\infty$ or $\infty-\infty=\infty$, respectively. Moreover, since $T_{P}$ is strictly increasing in both variables on $] 0,1]^{2}$, also the just constructed uninorm is strictly increasing on $] 0,1\left[{ }^{2}\right.$ in both variables.

(c) We can copy the construction from case (b), just taking right-open intervals instead of left-open ones. In this case we get a super-conorm and then the construction from Proposition 7 we get a uninorm.

Example 2 We can take a representable uninorm as the operation $*$. Let $h:[0,1] \rightarrow[-\infty, \infty]$ given by

$$
h(x)= \begin{cases}\ln 2 x & \text { for } x \leq \frac{1}{2} \\ -\ln (2(1-x)) & \text { for } x>\frac{1}{2}\end{cases}
$$

and consider the same system of intervals $I_{i}$ and transformations $f_{i}$ as in Example $1(\mathbf{b})$. Then we get a uninorm (either conjunctive or disjunctive) whose generator is

$\Gamma(i, x)= \begin{cases}\ln \left(2 f_{i}(x)\right) & \text { for } x \leq \frac{1}{2} \text { and } x \in I_{i}, \\ -\ln \left(2\left(1-f_{i}(x)\right)\right) & \text { for } x>\frac{1}{2} \text { and } x \in I_{i} .\end{cases}$

Since uninorms are strictly increasing neither on ] $0,1]^{2}$ nor on $\left[0,1\left[^{2}\right.\right.$, the operation constructed via paving from a uninorm $*$ cannot be strictly increasing on $] 0,1\left[^{2}\right.$.

\section{Conclusions}

In this paper we have introduced paving as a construction method for associative commutative and monotone operations if the basic 'paving stone' (operation $*$ ) possesses these three properties. Under some further conditions which were discussed in this paper, the resulting operation may have a neutral element. If $*$ is a generated operation, then the resulting operation is also generated, but the corresponding generator is a two-place function. In example 1 (b) we have constructed a nonrepresentable uninorm which is strictly increasing on $] 0,1\left[{ }^{2}\right.$ in both variables.

\section{Acknowledgements}

Martin Kalina has been supported from the VEGA grant agency, grant number 2/0059/12

This paper has been supported by the Project: Mobility - enhancing research, science and education at the Matej Bel University, ITMS code: 26110230082, under the Operational Program Education co-financed by the European Social Fund.

\section{References}

[1] S. Jenei, Structure of left-continuous triangular norms with strong induced negations, (II) Rotation-annihilation construction, Journal of Applied Non-Classical Logic, 11: 351-366, 2001.

[2] E. P Klement, R. Mesiar, and E. Pap, Quasiand pseudo-inverses of monotone functions, and the construction of t-norm, Fuzzy Sets and Systems, 104: 3-13, 1999.

[3] E. P Klement, R. Mesiar, and E. Pap, Triangular Norms, Springer, 1 edition, 2000.

[4] A. Mesiarová-Zemánková, Generated generalized uninorms and ordinal sum of uninorms, Int. J. Approx. Reasoning, submitted.

[5] B. Schweizer and A. Sklar, Probabilistic Metric Spaces, North Holland, New York, 1983.

[6] R. R. Yager and A. Rybalov, Uninorm aggregation operators, Fuzzy Sets and Systems, 80: 111-120, 1996. 